\title{
EFEITOS DO MÉTODO PILATES NO TORQUE ISOCINÉTICO DOS EXTENSORES E FLEXORES DO JOELHO: ESTUDO PILOTO
}

\author{
EFFECTS OF THE PILATES METHOD IN ISOKINETIC TORQUE OF KNEE EXTENSORS AND FLEXORS: \\ PILOT STUDY
}

\author{
EFECTOS DEL MÉTODO PILATES EN EL TORQUE ISOCINÉTICO DE LOS EXTENSORES Y FLEXORES DE \\ LA RODILLA: ESTUDIO PILOTO
}

\author{
Laís Campos de Oliveira \\ (Fisioterapeuta) \\ Deise Aparecida de Almeida Pires \\ Oliveira² (Fisioterapeuta) \\ Rodrigo Franco de Oliveira ${ }^{2}$ \\ (Fisioterapeuta) \\ Fabrício José Jassi' (Fisioterapeuta) \\ Fábio Antônio Néia Martini' \\ (Fisioterapeuta) \\ Raphael Gonçalves de Oliveira' \\ (Educador Físico) \\ 1. Universidade Estadual do Norte \\ do Paraná, Jacarezinho, PR, Brasil. \\ 2. Universidade Norte do Paraná, \\ Londrina, PR, Brasil.
}

\section{Correspondência:}

Centro de Ciências da Saúde Universidade Estadual do Norte do Paraná (UENP), Alameda Padre Magno, 841 - Nova Alcântara, Jacarezinho - PR, Brasil. 86.400-000 laispiraju@yahoo.com.br

\section{RESUMO}

Introdução: apesar da popularização do método Pilates como forma de exercício físico, os estudos com a técnica ainda não têm explorado os seus efeitos sobre o torque isocinético dos músculos extensores e flexores do joelho. Objetivo: verificar os efeitos do método Pilates no torque isocinético dos extensores e flexores do joelho em mulheres jovens. Métodos: 10 voluntárias foram submetidas à avaliação isocinética $(60 \%$ e $300 \%$ s) dos extensores e flexores do joelho, do membro inferior dominante, pré e pós-intervenção com o método Pilates, considerando-se o pico de torque (PT) e o trabalho total (TT). Oito intervenções foram realizadas ao longo de quatro semanas, constando de 28 exercícios de alongamento e fortalecimento para os principais grupos musculares. A análise estatística, utilizando os testes $t$ de Student ou Wilcoxon para amostras dependentes, foi utilizada $(p<0,05)$. Resultados: os resultados mostraram melhora significativa para a maioria das variáveis observadas, tanto na extensão do joelho (TT 60\% $/ \mathrm{s}-8,98 \%, \mathrm{p}=0,0166 ; \mathrm{PT} 300^{\circ} / \mathrm{s}-11,80 \%, \mathrm{p}=$ 0,$0077 ;$ TT $300 \%$ - 19,68\%, $p=0,0051$ ), quanto na flexão (PT 60\% $/ \mathrm{s}-11,44 \%, p=0,0171$; TT $60 \%$ s $-11,55 \%$, $\mathrm{p}=0,0395 ; \mathrm{TT} 300 \% \mathrm{~s}-12,86 \%, \mathrm{p}=0,0145)$, com exceção para duas variáveis, uma referente ao movimento de extensão do joelho (PT 60\% $/ \mathrm{s}-3,04 \%, \mathrm{p}=0,4413$ ) e outra ao movimento de flexão (PT $300 \% \mathrm{~s}-2,30 \%, \mathrm{p}=$ 0,3873). Conclusão: foi possível verificar que oito sessões de Pilates, realizadas ao longo de quatro semanas, proporcionaram melhora significativa do torque isocinético dos músculos extensores e flexores do joelho em mulheres jovens, em relação ao PT e TT $(60 \%$ s e $300 \%$ s) para a maioria das variáveis analisadas.

Palavras-chave: exercício, treinamento de resistência, força muscular.

\section{ABSTRACT}

Introduction: despite the popularity of Pilates as a form of exercise, studies with the technique still have not explored their effects on isokinetic torque of the extensor and flexor muscles of the knee. Objectives: check the effects of the Pilates method on isokinetic torque of the knee extensors and flexors in young women. Methods: 10 volunteers underwent isokinetic evaluation $(60 \%$ and $300 \%$ ) of the knee extensors and flexors of the dominant leg, pre-and post-intervention with the Pilates method, considering the peak torque (PT) and total work (TW). Eight interventions were conducted over four weeks, 28 consisting of stretching and strengthening exercises for the major muscle groups. Statistical analysis using the Student $t$ or Wilcoxon for dependent samples was used $(p<0.05)$. Results: the results showed significant improvement for most of the observed variables in both knee extension (TW 60\% $\%$ 8.98\%, $p=0.0166$, PT 300\% $-11.80 \%, p=0.0077$, TW $300 \% \mathrm{~s}-19.68 \%, p=0.0051$ ) and in flexion (PT 60\% $-11.44 \%, p=0.0171$; TW 60\% $/ 5-11.55 \%, p=0.0395$; TW $300 \%-12.86 \%, p=0.0145$ ), as an exception to two variables: one related to the movement of knee extension (PT $60 \% \mathrm{~s}-3.04 \%, p=0.4413$ ) and the other to flexion (PT 300\% $\mathrm{s}-2.30 \%, p=0.3873$ ). Conclusion: we found that eight sessions of Pilates, conducted over four weeks, provided significant improvement in isokinetic torque of the extensor and flexor muscles of the knee in young women, in relation to the PT and TT $(60 \%$ and $300 \%)$ for most variables.

Keywords: exercise; resistance training; muscle strength.

\section{RESUMEN}

Introducción: a pesar de la popularización del método Pilates, como una forma de ejercicio físico, los estudios con la técnica aún no han explorado sus efectos sobre el torque isocinético de los músculos extensores y flexores de la rodilla. Objetivos: verificar los efectos del método Pilates, el torque isocinético de los extensores y flexores de la rodilla en mujeres jóvenes. Métodos: diez voluntarias fueron sometidas a evaluación isocinética (60\% y $300 \%$ s) de los extensores y flexores de la rodilla, del miembro inferior dominante, antes y después de la intervención con el método Pilates, considerándose el pico de torque (PT) y trabajo total (TT). Ocho intervenciones fueron realizadas durante cuatro semanas, siendo 28 ejercicios de alongamiento y fortalecimiento para los principales grupos mus- 
culares. Fue aplicado análisis estadístico utilizando los tests t de Student o de Wilcoxon para muestras dependientes $(p<0,05)$. Resultados: Ios resultados mostraron mejora significativa para la mayoría de las variables observadas, tanto en extensión de la rodilla (TT 60\% $\%$ 8,98\%, $p=0,0166$, PT 300\% $/ \mathrm{s}-11,80 \%, p=0,0077$, TT 300\% $\mathrm{s}-19,68 \%$, $p=0,0051$ ) como en la flexión (PT 60\% $/ \mathrm{s}-11,44 \%, p=0,0171 ;$ TT 60\% $/ \mathrm{s}-11,55 \%, p=0,0395 ;$ TT 300\% $/ \mathrm{s}-12,86 \%, p$ = 0,0145), como excepción a dos variables: una referente al movimiento de extensión de la rodilla (PT 60\% $\%$ - 3,04 $\%, p=0,4413$ ) yotra al movimiento de flexión (PT 300\%s - 2,30\%, p = 0,3873). Conclusión: fue posible verificar que ocho sesiones de Pilates, realizadas a lo largo de cuatro semanas, proporcionó mejora significativa en el torque isocinético, de los músculos extensores y flexores de la rodilla en mujeres jóvenes, en relación al PT y TT $(60 \%$ y $300 \%$ s), para la mayoría de las variables analizadas.

Palabras clave: ejercicio, entrenamiento de resistencia, fuerza muscular.

\section{INTRODUÇÃO}

O método Pilates é uma modalidade de exercício físico idealizada pelo alemão Joseph Hubertus Pilates, no período da Primeira Guerra Mundial. Recentemente a técnica vem ganhando cada vez mais adeptos no mundo todo. Somente nos Estados Unidos (país precursor do método) havia mais de cinco milhões de praticantes no início deste século, sendo a sétima modalidade com maior número de adeptos naquele país, entre os anos de 2008 e $20099^{1}$.

No entanto, os estudos sobre a técnica ainda são escassos, se restringindo principalmente, aos potenciais efeitos sobre a estabilização lombo-pélvica e flexibilidade, como forma de prevenção ou tratamento da dor lombar ${ }^{2-5}$. Apesar do método também preconizar o fortalecimento de outras regiões do corpo, como a musculatura de membros superiores e inferiores, pouco se sabe sobre como o Pilates atua na força muscular destes grupos ${ }^{1,5}$.

Para os membros inferiores em específico, o nível de fortalecimento muscular pode contribuir na execução de tarefas do cotidiano ${ }^{6}$, ou representar ganho de desempenho em diversas modalidades esportivas $^{7,8}$. Os principais grupos musculares desta região são os envolvidos na tarefa de extensão e flexão do joelho ${ }^{6}$.

Dentre as formas de treinamento físico, capazes de proporcionar aumento da força destes músculos, estão aqueles que fazem o uso da resistência com pesos. Pessoas saudáveis ${ }^{9}$, atletas ${ }^{10}$, ou em processo de reabilitação ${ }^{11}$ utilizam-se desta forma de exercício, objetivando aumento da força muscular dos extensores e flexores do joelho ${ }^{9-11}$.

Como o método Pilates é uma forma de exercício físico, que começou a ser pesquisado com mais frequência apenas recentemente, não existem evidências, quanto ao seu potencial efeito, sobre variáveis relacionadas ao fortalecimento muscular dos extensores e flexores do joelho em população adulta jovem. O objetivo do presente trabalho, foi verificar os efeitos do método Pilates, no torque isocinético dos extensores e flexores do joelho, em um grupo de mulheres jovens, submetidas a um curto período de treinamento.

\section{MATERIAIS E MÉTODOS}

Uma amostra de conveniência foi utilizada para este estudo, que se caracterizou do tipo quase-experimental, com um grupo pré e pós-teste ${ }^{12}$. Foram convidadas a participar, estudantes universitárias cursando o último ano, de um curso de graduação, em uma universidade estadual paranaense. O recrutamento ocorreu no mês de outubro de 2013. Todas as acadêmicas que demonstraram interesse e atenderam aos critérios de inclusão, fizeram parte da amostra.

Os critérios de inclusão foram: a) idade entre 20 e 30 anos; b) sexo feminino; c) índice de massa corporal (IMC) dentro dos parâmetros normais (entre 18,5 e 24,9 Kg/m²); d) apresentação de atestado médico indicando aptidão para a prática de exercícios físicos; e) não estarem praticando nenhum tipo de exercício físico por pelo menos seis meses; f) concordarem em não praticar outro tipo de exercício físico durante a pesquisa.

Os critérios de exclusão foram: a) limitações funcionais para deambular ou o uso de dispositivos de apoio (bengala e muletas); b) resposta afirmativa em qualquer pergunta de um questionário para identificar comorbidades relacionadas a problemas visuais graves, cirurgias ortopédicas de quadril, joelhos ou tornozelos, ou queixa de dor relacionada à região de membros inferiores.

Atenderam aos critérios 10 voluntárias, com idades entre 21 e 27 anos $(22,5 \pm 1,80)$, peso corporal entre 44,7 e $71 \mathrm{Kg}(57,76 \pm 7,53)$, altura entre 152 e $174 \mathrm{~cm}(161,1 \pm 6,12)$ e IMC entre 18,61 e $24,9 \mathrm{Kg} / \mathrm{m}^{2}(22,19 \pm 2,03)$. Todas assinaram Termo de Consentimento Livre e Esclarecido, após serem devidamente informadas sobre os objetivos e metodologia do estudo, que foi aprovado por Comitê de Ética local através do parecer 49/10.

A avaliação isocinética da musculatura extensora e flexora do joelho foi realizada com o dinamômetro isocinético Biodex System 4.0 (Biodex Medical Systems, Shirley, NY, EUA), pré e pós-intervenção.

Antecedendo ao teste, o aparelho foi calibrado seguindo as normas do fabricante. $\mathrm{O}$ aquecimento foi realizado durante $5 \mathrm{~min}$ em bicicleta ergométrica vertical, com carga leve (25 Watts) e velocidade confortável (50 rotações por minuto). Na sequência, o avaliador explicou detalhadamente os procedimentos do teste e posicionou as voluntárias no assento do equipamento, com o ângulo da articulação do quadril em 120 graus de flexão. $O$ eixo de rotação do braço do dinamômetro foi alinhado com o epicôndilo lateral do fêmur do membro inferior direito (relatado como dominante por todas as voluntárias). 0 local da aplicação da força foi posicionado, aproximadamente, dois centímetros do maléolo medial. Foram fixados cintos no tronco, pelve e coxa, a fim de evitar movimentos compensatórios ${ }^{13}$. Após uma breve familiarização com o equipamento e com a amplitude de movimento, a voluntária realizou o teste de extensão e flexão do joelho (concêntrico/concêntrico) na velocidade de 60 graus por segundo $\left(60^{\circ} / \mathrm{s}\right)$, partindo de uma posição em que a articulação do joelho se encontrava em 90 graus de flexão. Foram realizadas três séries de cinco repetições, com 30 segundos de descanso entre as séries. Em seguida o teste foi realizado seguindo o mesmo protocolo, na velocidade de 300 graus por segundo $(300 \%$ s). As variáveis consideradas para o estudo foram o pico de torque (PT) e o trabalho total (TT), expressos em Newtons por metro $(\mathrm{Nm})$ e joules $(\mathrm{J})$ respectivamente.

As voluntárias foram instruídas a realizar a máxima força de contração voluntária dos extensores do joelho, seguida imediatamente pela contração máxima dos flexores do joelho, de forma contínua. Durante o teste foram usadas palavras de incentivo, como "mais força" e "mais forte". O procedimento foi realizado pelo mesmo avaliador, pré e pós-intervenção, com experiência em avaliação isocinética. 


\section{Protocolo de Intervenção}

As intervenções constaram de oito sessões de Pilates realizadas duas vezes por semana, durante quatro semanas. Cada intervenção teve duração de 60 minutos. Entre uma sessão e outra, foi respeitado um intervalo de no mínimo dois dias. Antes de iniciar as intervenções, as voluntárias realizaram uma sessão de familiarização com o Pilates, na qual foi demonstrada a execução correta de cada movimento, assim como, ocorreu a explicação dos princípios do método.

Os equipamentos utilizados para a realização dos exercícios foram: Cadeira Combo, Cadillac Trapézio, Reformer Universal, Ladder Barrel e Wall Unit, todos da linha de equipamentos para Pilates do Instituto São Paulo (Instituto de Ortopedia e Fisioterapia São Paulo, Brasil), registrados na Agência Nacional de Vigilância Sanitária (ANVISA, Brasil). Também foram realizados exercícios no solo, sobre um colchonete (Mat), sem o uso de acessórios.

Foram selecionados para a presente pesquisa, 28 exercícios de fortalecimento e alongamento, envolvendo os principais grupos musculares. A sequência de realização dos exercícios, os equipamentos utilizados e o nome tradicional de cada movimento do método Pilates, foram respectivamente:

a) alongamentos iniciais no Reformer Universal (Long Stretches Elephant; Front Splits; Arabesche; Splits With Control; Stomach Massage Series);

b) fortalecimento dos membros inferiores na Cadeira Combo (Going Up Front; Footwork Double Leg Pumps; Footwork Double Leg Pumps Switching) e no Cadillac Trapézio (Leg Series Supine Lowers; Leg Series Supine Scissors; Tower);

c) fortalecimento dos flexores do tronco no Cadillac Trapézio (Sit Up; Sit Up One Leg) e Mat (The Hundred; Double Leg Stretch; Criss Cross; Teaser);

d) fortalecimento dos extensores do tronco no Ladder Barrel (Trunk Extension; Leg Extension);

e) fortalecimento dos membros superiores no Wall Unit (Arms Pulling; Pulling the Chest; Arms Biceps; Arms Triceps);

F) alongamentos finais no Ladder Barrel (Stretches Back; Stretches Front; Stretches the Gluteus; Stretches to the Side; Stretch Back and Forward).

Todos os exercícios foram realizados em uma série de dez repetições. A intensidade das molas foi substituída (mudando a posição das mesmas nos equipamentos, ou substituindo-as por outras de maior intensidade) conforme a evolução da força das voluntárias, mantendo-se o número de repetições e série. Para determinar o nível de esforço e consequentemente a evolução das cargas, foram utilizadas descrições verbais de acordo com a escala de Borg CR1014. carga leve (Borg $\leq 2)$, carga moderada (Borg $>2-<5$ ), carga pesada (Borg $\geq 5-<7$ ) e próximo da carga máxima (Borg $\geq 7$ ). O nível de esforço mantido durante as sessões foi pesado (Borg entre 5 e 6). Todas as vezes que a intensidade do exercício foi alterada, a nova carga utilizada era imediatamente anotada em uma ficha individual, utilizada para registro do treinamento.

Os exercícios foram selecionados na tentativa de melhorar a flexibilidade e a força muscular globalmente, como tradicionalmente é trabalhado no método Pilates. As voluntárias foram orientadas sobre os princípios do método (Centro, Controle, Concentração, Fluidez, Precisão e Respiração) que precisavam ser respeitados na execução de cada exercício'.

As intervenções foram ministradas por um profissional com certificação em Pilates e experiência com o método.

\section{Análise Estatística}

Foi realizada análise descritiva dos dados, expressos na forma de média e desvio padrão. Para verificar a normalidade da distribuição dos dados, foi realizado o teste Shapiro-Wilk. Os momentos pré e pós-intervenção foram comparados através dos testes $t$ de Student e Wilcoxon, para amostras dependentes, quando os dados apresenta- vam distribuição paramétrica ou não paramétrica, respectivamente. O intervalo de confiança admitido em todos os testes foi de $95 \%$ $(p<0,05)$. Os dados foram tratados no programa SPSS 20.0 (SPSS Corp., Chicago, IL, EUA).

\section{RESULTADOS}

No movimento de extensão do joelho, ocorreu aumento significativo para a maioria dos parâmetros analisados (TT 60\% $/ \mathrm{s}, 8,98 \%, \mathrm{p}=$ 0,0166; PT 300\% $/$ - 11,80\%, p =0,0077; TT 300\% $/$ - 19,68\%, p=0,0051), em relação ao período pré-intervenção (tabela 1), assim como, para os flexores do joelho (PT 60\% $/ \mathrm{s}-11,44 \%, \mathrm{p}=0,0171$; TT $60 \%$ s $-11,55 \%, \mathrm{p}=$ 0,$0395 ;$ TT $300 \%$ - 12,86\%, $p=0,0145$ ) (tabela 2). Não houve diferença significativa em dois parâmetros avaliados; um referente ao movimento de extensão do joelho (PT 60\% $/$ - 3,04\%, p = 0,4413) (tabela 1) e outro, ao movimento de flexão (PT 300\% - 2,30\%, p =0,3873) (tabela 2).

Tabela 1. Determinação do pico de torque (PT) e trabalho total (TT), no teste isocinético realizado a 60 e 300 graus de velocidade angular $(60 \%$ s e $300 \%$ s), sobre os extensores do joelho, do membro inferior dominante.

\begin{tabular}{c|c|c|c}
\hline Avaliação & Pré-intervenção & Pós-intervenção & $\mathbf{p}$ \\
\hline PT $(\mathrm{Nm}) 60^{\circ} / \mathrm{s}$ & $129,27 \pm 19,23$ & $133,20 \pm 19,46$ & 0,4413 \\
\hline $\mathrm{TT}(\mathrm{J}) 60^{\circ} / \mathrm{s}$ & $565,20 \pm 74,15$ & $615,93 \pm 98,45^{*}$ & 0,0166 \\
\hline $\mathrm{PT}(\mathrm{Nm}) 300^{\circ} / \mathrm{s}$ & $66,12 \pm 14,62$ & $73,92 \pm 18,65^{*}$ & 0,0077 \\
\hline $\mathrm{TT}(\mathrm{J}) 300^{\circ} \mathrm{s}$ & $331,56 \pm 77,64$ & $396,82 \pm 93,94^{*}$ & 0,0051 \\
\hline
\end{tabular}

* diferença estatística em relação à situação pré-intervenção $(p<0,05)$. Nm = newtons por metro; J = joules.

Tabela 2. Determinação do pico de torque (PT) e trabalho total (TT), no teste isocinético realizado a 60 e 300 graus de velocidade angular $(60 \%$ s e $300 \%$ s), sobre os flexores do joelho, do membro inferior dominante.

\begin{tabular}{c|c|c|c}
\hline Avaliação & Pré-intervenção & Pós-intervenção & $\mathbf{p}$ \\
\hline $\mathrm{PT}(\mathrm{Nm}) 60^{\circ} / \mathrm{s}$ & $62,40 \pm 9,88$ & $69,54 \pm 12,94^{*}$ & 0,0171 \\
\hline $\mathrm{TT}(\mathrm{J}) 60^{\circ} / \mathrm{s}$ & $358,27 \pm 77,36$ & $399,65 \pm 75,68^{*}$ & 0,0395 \\
\hline $\mathrm{PT}(\mathrm{Nm}) 300^{\circ} / \mathrm{s}$ & $49,16 \pm 9,68$ & $50,29 \pm 9,07$ & 0,3873 \\
\hline TT $(\mathrm{J}) 300^{\circ} / \mathrm{s}$ & $231,34 \pm 72,21$ & $261,08 \pm 50,77^{*}$ & 0,0145 \\
\hline
\end{tabular}

\section{DISCUSSÃO}

Mesmo existindo uma crescente procura pelo método Pilates, enquanto forma de condicionamento físico, ou mesmo para reabilitação fisioterapêutica' ${ }^{\prime}$ até aonde é de conhecimento dos autores da presente pesquisa, nenhum estudo foi encontrado, com o intuito de verificar o torque isocinético dos extensores e flexores do joelho, em pessoas submetidas a esta prática. O dinamômetro isocinético, é um equipamento que fornece informações precisas nas avaliações relacionadas a estes grupos musculares, com excelente confiabilidade teste-reteste, principalmente quando se utiliza o TT como variável ${ }^{15}$.

Para a musculatura extensora e flexora do joelho, foi possível observar uma melhora significativa para o TT, tanto em uma velocidade angular mais baixa $\left(60^{\circ} / \mathrm{s}\right)$, quanto em uma velocidade angular mais alta $(300 \%$ s). O PT (que se refere ao ponto de maior torque na amplitude de movimento), se alterou significativamente (pré e pós-intervenção) para o movimento de extensão $\left(300^{\circ}\right.$ /s) e flexão $\left(60^{\circ}\right.$ s), em uma velocidade mais alta e baixa, respectivamente, apresentando melhora pouco significativa para as outras duas variáveis (extensão à $60 \%$ s e flexão à $300^{\circ} / \mathrm{s}$ ).

A não melhora significativa para estas duas últimas variáveis, pode ter sido influenciada, pelo curto período de intervenção (oito sessões, durante quatro semanas). Programas de treinamento físico de resistência muscular, que tiveram como objetivo identificar o PT isocinético dos extensores e flexores do joelho em mulheres jovens, tipicamente se utilizam de períodos de intervenção maiores (superiores a 10 semanas), para identificar melhora estatisticamente significante ${ }^{16,17}$. 
Mesmo com um curto período de intervenção, a maior parte das variáveis analisadas apresentaram diferença significativa. Provavelmente, um fator determinante, para que em pouco tempo houvesse meIhora do torque isocinético, foi o controle da carga de treinamento (que era anotada em uma ficha individual de acompanhamento), assim como, o nível de esforço, que se manteve dentro de uma intensidade pesada, conforme a escala de percepção do esforço ${ }^{14}$.

Como a amostra de voluntárias do presente artigo, não foi composta por indivíduos em processo de reabilitação, mas sim, jovens assintomáticas e saudáveis, foi possível trabalhar dentro de uma zona mais intensa de treinamento. Neste caso, levou-se em consideração, que as molas apresentam torque de resistência que se modificam no momento em que estas se deformam durante a execução do exercício, dependendo também, do posicionamento que ocupam no equipamento ou da sua intensidade (que é distinguida por cores) ${ }^{18,19}$.

A maior parte das pesquisas com o método Pilates, concentram-se nos efeitos da técnica no fortalecimento da região lombo-pélvica, como possível contribuinte na melhora da dor lombar. Nestes casos, o controle de intensidade e nível do esforço, são tipicamente realizados de maneira subjetiva, pelo profissional que ministra a prática (no momento em que o executante realiza um determinado movimento, fica a cargo do profissional, identificar se o exercício está sendo realizado corretamente, ou seja, sem a existência de compensações posturais, o que possibilitaria o aumento do nível de esforço) ${ }^{20-22}$.

Em pesquisas cujo o objetivo seja a melhora da força muscular, principalmente em casos de condicionamento físico, um controle menos subjetivo do nível de esforço pode ser determinante. Tradicionalmente na prática do método Pilates isto não acontece, provavelmente porque a maioria das pesquisas, tem se dedicado a verificar os efeitos da técnica, em casos de reabilitação fisioterapêutica. No entanto, este controle se mostra efetivo e fundamental para ganho de performance em indivíduos saudáveis ${ }^{23}$ ou para melhora do desempenho esportivo ${ }^{24}$.

As pesquisas com o método Pilates precisam aprofundar os conhecimentos sobre esta variável (controle do nível de intensidade do treinamento), além de outras, como periodização do treino e os diferentes protocolos de intervenção, que podem ser utilizados para melhora do desempenho, tanto de não atletas, como também, nas mais diversas modalidades esportivas.

Para estudos posteriores, sugere-se a aplicação do método em indivíduos treinados, no contexto de práticas esportivas, de forma a identificar os efeitos da técnica, sobre o torque isocinético dos extensores e flexores do joelho; ajudando desta forma, a identificar se o Pilates pode ser utilizado não somente na reabilitação ${ }^{5}$, mas também como coadjuvante no condicionamento físico de atletas.

A utilização de uma amostra de conveniência e a não randomização, com formação de um grupo controle, deve ser considerada como limitação da presente pesquisa. Espera-se que este estudo, possa contribuir com a discussão sobre a temática e suscitar indagações para futuras pesquisas, uma vez que inexistem estudos prévios, envolvendo a variável aqui explorada.

\section{CONCLUSÃO}

Um protocolo de exercícios do método Pilates, realizado duas vezes por semana, durante quatro semanas, foi capaz de proporcionar melhora significativa do torque isocinético, dos músculos envolvidos na tarefa de extensão e flexão do joelho em mulheres jovens, para à maioria das variáveis referentes ao pico de torque e ao trabalho total, à 60 e 300 graus por segundo.

Todos os autores declararam não haver qualquer potencial conflito de interesses referente a este artigo.

\section{REFERÊNCIAS}

1. Di Lorenzo CE. Pilates: what is it? Should it be used in rehabilitation? Sports Health. 2011;3(4):352-61.

2. Rossi DM, Morcelli MH, Marques NR, Hallal CZ, Gonçalves M, Laroche DP, Navega MT. Antagonist coactivation of trunk stabilizer muscles during Pilates exercises. J Bodyw Mov Ther. 2014;18(1):34-41.

3. Phrompaet S, Paungmali A, Pirunsan U, Sitilertpisan P. Effects of pilates training on lumbo-pelvic stability and flexibility. Asian J Sports Med. 2011;2(1):16-22.

4. Wells C, Kolt GS, Bialocerkowski A. Defining Pilates exercise: a systematic review. Complement Ther Med. 2012;20(4):253-62.

5. Kloubec J. Pilates: how does it work and who needs it? Muscles Ligaments Tendons J. 2011;29(2):61-6.

6. D'Lima DD, Fregly BJ, Patil S, Steklov N, Colwell CW. Knee joint forces: prediction, measurement, and significance. Proc Inst Mech Eng H. 2012; 226(2):95-102.

7. Zabka FF, Valente HG, Pacheco AM. Isokinetic evaluation of knee extensor and flexor muscles in professional soccer players. Rev Bras Med Esporte. 2011;17(3):189-92.

8. Lockie RG, Schultz AB, Callaghan SJ, Jeffriess MD. The effects of isokinetic knee extensor and flexor strength on dynamic stability as measured by functional reaching. Isokinet Exerc Sci. 2013;21(4):301-9.

9. Souza Júnior TP, Fleck SJ, Simão R, Dubas JP, Pereira B, Pacheco EM, et al. Comparison between constant and decreasing rest intervals: influence on maximal strength and hypertrophy. J Strength Cond Res. 2010;24(7):1843-50

10. Day B, Johnson D. Strength and conditioning for triathletes. Sports Med Arthrosc. 2012;20(4):225-30.

11. Augustsson J. Documentation of strength training for research purposes after $A C L$ reconstruction. Knee Surg Sports Traumatol Arthrosc. 2013;21(8):1849-55.

12. Page P. Research designs in sports physical therapy. Int J Sports Phys Ther. 2012; 7(5):482-92.

13. Stumbo TA, Merriam S, Nies K, Smith A, Spurgeon D, Weir JP. The effect of hand-grip stabilization on isokinetic torque at the knee. J Strength Cond Res. 2001;15(3):372-7.

14. Borg GAV. Psychophysical bases of perceived exertion. Med Sci Sports Exerc. 1982;14(5):377-81.
15. Saenz A, Avellanet M, Hijos E, Chaler J, Garreta R, Pujol E, et al. Knee isokinetic test-retest: a multicentre knee isokinetic test-retest study of a fatigue protocol. Eur J Phys Rehabil Med. 2010;46(1):81-8.

16. Silva RF, Cadore EL, Kothe G, Guedes M, Alberton CL, Pinto SS, et al. Concurrent training with different aerobic exercises. Int J Sports Med. 2012;33(8):627-34.

17. Dannelly BD, Otey SC, Croy T, Harrison B, Rynders CA, Hertel JN, et al The effectiveness of traditional and sling exercise strength training in women. J Strength Cond Res. 2011;25(2):464-71.

18. Melo MO, Gomes LE, Silva YO, Bonezi A, Loss JF. Assessment of resistance torque and resultant muscular force during Pilates hip extension exercise and its implications to prescription and progression. Braz J Phys Ther. 2011;15(1):23-30.

19. Silva YO, Melo MO, Gomes LE, Bonezi A, Loss JF. Analysis of the external resistance and electromyographic activity of the hip extension performed according to the Pilates method. Braz J Phys Ther 2009;13(1):82-8.

20. da Luz MA Jr, Costa LO, Fuhro FF, Manzoni AC, Oliveira NT, Cabral CM. Effectiveness of mat pilates or equipment-based pilates exercises in patients with chronic nonspecific low back pain: a randomized controlled trial. Phys Ther. 2014;94(5):623-31.

21. Miyamoto GC, Costa LO, Galvanin T, Cabral CM. Efficacy of the addition of modified pilates exercises to a minimal intervention in patients with chronic low back pain: a randomized controlled trial. Phys Ther. 2013;93(3):310-20.

22. Miyamoto GC, Costa LO, Galvanin T, Cabral CM. The efficacy of the addition of the Pilates method over a minimal intervention in the treatment of chronic nonspecific low back pain: a study protocol of a randomized controlled trial. J Chiropr Med. 2011;10(4):248-54.

23. Shoepe TC, Ramirez DA, Rovetti RJ, Kohler DR, Almstedt HC. The effects of 24 weeks of resistance training with simultaneous elastic and free weight loading on muscular performance of novice lifters. J Hum Kinet. 2011;29:93-106.

24. Bartolomei S1, Hoffman JR, Merni F, Stout JR. A comparison of traditional and block periodized strength training programs in trained athletes. J Strength Cond Res. 2014;28(4):990-7. 